\title{
The influence of total and directional energy of late sound on listener envelopment
}

\author{
Hiroshi Furuya ${ }^{1, *}$, Kazutoshi Fujimoto ${ }^{2}$, Akiko Wakuda ${ }^{3}$ and Yusuke Nakano ${ }^{3}$ \\ ${ }^{1}$ Department of Architecture, Faculty of Engineering, Kyushu Kyoritsu University \\ ${ }^{2}$ Department of Architecture and Urban Design, Faculty of Human-Environment Studies, Kyushu University \\ ${ }^{3}$ Department of Architecture, Graduate School of Human-Environment Studies, Kyushu University
}

(Received 30 August 2004, Accepted for publication 23 September 2004)

Keywords: Spatial impression, Directional late sound, Psychological experiment, Concert hall acoustics PACS number: 43.55.Hy [DOI: 10.1250/ast.26.208]

\section{Introduction}

In concert hall acoustics, spatial perception is one of the most important psychological factors that aid in the evaluation of the sound field. The purpose of this study is to clarify the relation between subjective listener envelopment $(L E V)$ and directional characteristics of late-arriving sound through a series of psycho-acoustical experiments. In our previous works $[1,2]$, it was found that late sounds from above and behind the listener, as well as lateral, affected $L E V$ significantly when the late-to-early energy ratio $T L L$ was constant, and that late overhead and back energy ratios were definitely effective for $L E V$ at the rate of around fifty percent of the effect of lateral energy ratio. Those experiments were designed on the condition that all of the late sound had a single directional energy component, namely that the arrival directions were restricted to lateral, frontal, overhead, and back, in order to examine directly the effects of fundamental directional energy components.

In this paper, two kinds of psycho-acoustical experiments are conducted in order: (1) to investigate whether or not the effect of late sounds with a single directional energy component on $L E V$ is applicable to the general case when the late sounds have plural directional components, namely coming from the oblique direction, and (2) to clarify the degrees of contribution of directional late energy ratio $(D L R)$ and total late energy on $L E V$ by using sound fields in which both $D L R$ and TLL are varied.

\section{Method}

Subjective experiments were conducted using simulated sound fields in an anechoic chamber. The structure of the sound fields is shown in Fig. 1. The sound fields consisted of a direct sound, six discrete early reflections derived from multitap delay machines, and later sound added by digital reverberators. In all tests, a loudspeaker for direct sound and two loudspeakers for early reflections were fixed, and the delay times and the levels of early reflections relative to the direct sound were kept constant so that $L F_{80}$ was 0.17 . Late sound arriving more than $80 \mathrm{~ms}$ after the direct sound was fed to six or five loudspeakers, respectively, corresponding to Expeiments 1 and 2. All the loudspeakers were equidistant $(1.5 \mathrm{~m})$ from the listener. The reverberation time was set at $1.8 \mathrm{~s}$.

\footnotetext{
*e-mail: hifuruya@kyukyo-u.ac.jp
}

A method of paired comparisons was employed for all tests. An anechoic recording of the $10 \mathrm{~s}$ section of Bizet's 'L'Arlesienne, Suite no. 2, Menuetto' (bars 15-18) was used as the music motif. All the sound field pairs were presented to the subjects in random order. The subjects were students, 20 to 25 years old, with normal hearing sensitivity. Before the experiments, the term 'listener envelopment' was explained to them using a conceptual illustration and some comments, which expressed the definition of $L E V$. A preliminary practice session was held in order to ensure that the subjects were familiar with the requirements of each test. Each subject was individually required to judge whether the $L E V$ for the second stimulus was weaker or stronger than that for the preceding one in a pair of sound fields. Psychological interval scales of $L E V$ were constructed from the experimental results by the method in Thurstone's Case V.

Four DLRs were calculated from the overall impulse responses obtained with omni-directional and figure-of-eight microphones in order to determine the directional property of late sound. Late lateral energy ratio $L E_{\text {late }}$, late frontal energy

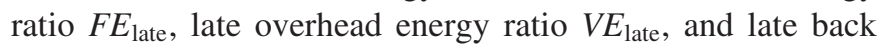
energy ratio $B E_{\text {late }}$ were defined as ratios of each directional late energy to the total late energy. The late-to-early energy ratio $T L L$ was defined as a relative level of the late energy to the early one, which was the reciprocal of $C_{80}$. The Aweighted binaural SPL [3], BSPL, was measured as an indicator of loudness, using a dummy head microphone for the music source. The $B S P L$ was constant at $63 \mathrm{~dB}$ in all tests.

\section{Effect of late sound with plural directional energy components on $\boldsymbol{L E V}$}

In Experiment 1, the effect of DLR on $L E V$ is examined using sound fields with late sounds arriving from oblique directions in order to clarify whether or not the degrees of contribution of late energy with a single directional component is valid when the late sounds have plural directional energy components.

\subsection{Experimental conditions}

Figure 2 shows the arrangement of the loudspeakers. Six loudspeakers were located for late sounds. The late sounds except from behind the listener consisted of two or three directional energy components. The three $D L R \mathrm{~s}, L E_{\text {late }}, V E_{\text {late }}$, and $B E_{\text {late }}$, were varied keeping the $T L L$ constant. The frontal

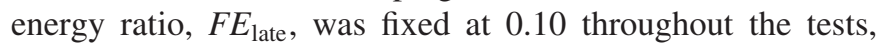
because the effect of late frontal sound on $L E V$ was found to 
Direct sound

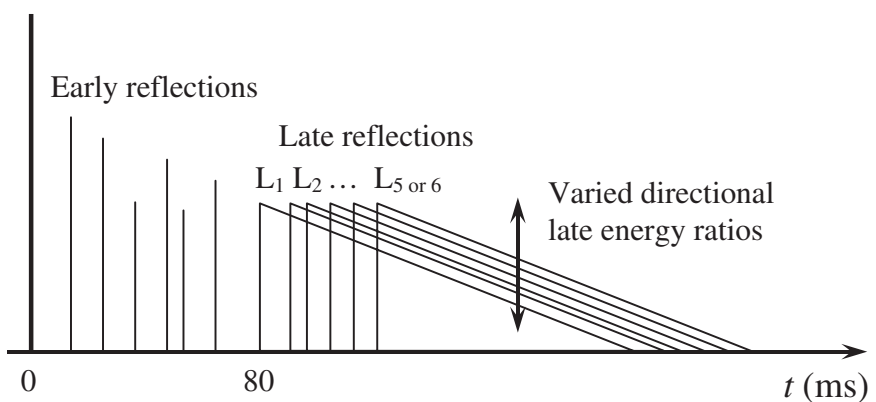

Table 1 Seven sound fields used in Experiment 1.

\begin{tabular}{cccccc}
\hline \multirow{2}{*}{$\begin{array}{c}\text { Stimulus } \\
\text { no. }\end{array}$} & \multirow{2}{*}{$B S P L$} & \multicolumn{4}{c}{ Directional late energy ratios } \\
\cline { 3 - 6 } & $(\mathrm{dB})$ & $L E_{\text {late }}$ & $V E_{\text {late }}$ & $B E_{\text {late }}$ & $F E_{\text {late }}$ \\
\hline 1 & & 0.30 & 0.30 & 0.30 & 0.10 \\
2 & & 0.20 & 0.50 & 0.20 & 0.10 \\
3 & & 0.30 & 0.10 & 0.50 & 0.10 \\
4 & \multirow{2}{*}{63} & 0.50 & 0.25 & 0.15 & 0.10 \\
5 & & 0.10 & 0.25 & 0.55 & 0.10 \\
6 & & 0.60 & 0.10 & 0.20 & 0.10 \\
7 & & 0.20 & 0.55 & 0.15 & 0.10 \\
\hline
\end{tabular}

Fig. 1 Structure of the sound fields used in the experiments. In Experiment 1, late sound consists of plural directional energy components, and the directional late energy ratios are varied keeping late-to-early energy ratio constant. In Experiment 2, the late-to-early energy ratio is also varied. Late sound was fed to six loudspeakers in Experiment 1, and to five in Experiment 2 .

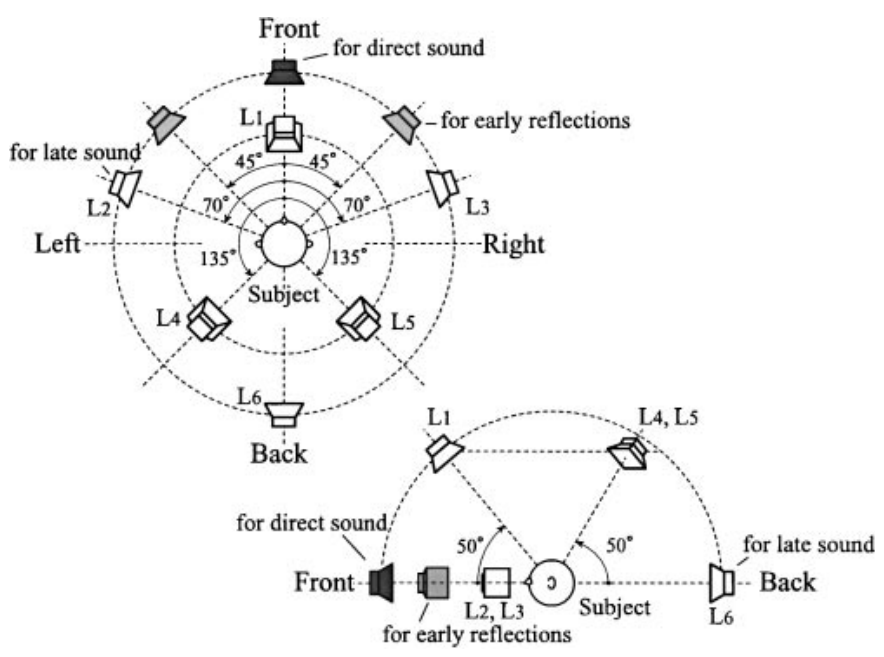

Fig. 2 Arrangement of loudspeakers in Experiment 1.

be much smaller than the others [2]. The experiment consisted of two separate tests, according to the values of TLL which were set at +3 and $0 \mathrm{~dB}$. As given in Table 1, seven kinds of sound fields were used in each test. All combinations of seven stimuli, twenty-one pairs, were presented to seven subjects. Each subject was tested individually to judge each pair of stimuli eight times, and thus, a total of 168 judgments were made.

\subsection{Results and discussion}

The results of conformity-tests with the Thurstone's Case $\mathrm{V}$ model showed that the experimental data was significant at a level below $1 \%$. The standard of judgment was agreed upon by all subjects at a level below $5 \%$ of significance.

The psychological interval scales for $L E V$ were obtained from the results, according to $T L L$ values of +3 and $0 \mathrm{~dB}$. Figure 3 shows the comparison between the measured $L E V$ in this experiment and calculated $L E V$ by using the regression equations that have been obtained from the previous experi-

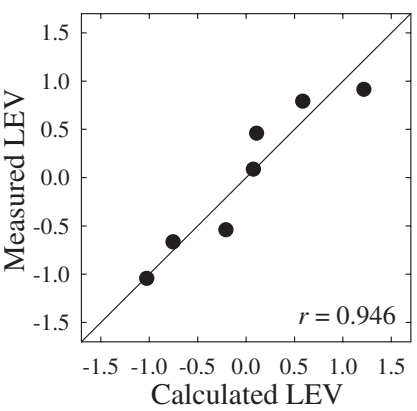

(a) $T L L=3 \mathrm{~dB}$

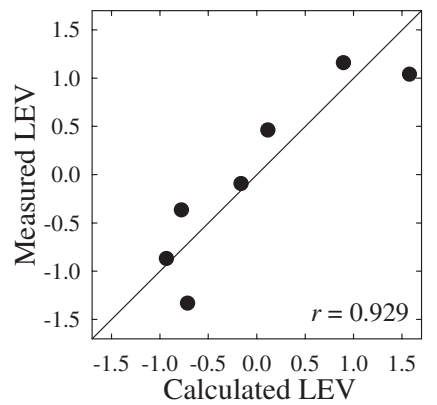

(b) $T L L=0 \mathrm{~dB}$
Fig. 3 Comparison between measured $L E V$ in Experiment 1 and calculated $L E V$ by the regression equation, which was obtained from the previous experiment [2] with late sounds having a single directional energy component: $r$, correlation coefficient.

Table 2 The results of multiple regression analyses between $L E V$ and directional late energy ratios in

\begin{tabular}{ccccc}
\hline \multirow{2}{*}{$T L L(\mathrm{~dB})$} & \multirow{2}{*}{$\begin{array}{c}\text { Multiple } \\
\text { correlation } \\
\text { coefficient }\end{array}$} & \multicolumn{3}{c}{ Standard regression coefficients } \\
\cline { 3 - 5 } & & $L E_{\text {late }}$ & $V E_{\text {late }}$ & $B E_{\text {late }}$ \\
\hline 3 & 0.980 & 1.558 & 0.433 & 0.783 \\
0 & 0.910 & 1.686 & 0.739 & 1.012 \\
\hline
\end{tabular}

ment [2] (named Experiment 0), in which all of the late sound had a single directional energy component. They give a good agreement, because the correlation coefficients between them are about 0.93 or more for any TLL. Additionally, multiple regression analyses were done for two conditions of TLL to investigate the degrees of contribution of $D L R$ to $L E V$, since the relation between the $D L R$ s and perceived $L E V$ could not be interpreted only with one directional parameter when the three $D L R$ s were varied simultaneously in each sound field. criterion variable, and $L E_{\text {late }}, V E_{\text {late }}$, and $B E_{\text {late }}$ as explanatory variables. Table 2 shows the results of analyses. The standard regression coefficient of $L E_{\text {late }}$ is the highest for any $T L L$. The coefficients of $V E_{\text {late }}$ and $B E_{\text {late }}$ are approximately thirty to Experiment 1, significant at $p<0.005$. The psychological interval scale for $L E V$ was used as a 
sixty percents of that of $L E_{\text {late }}$. This tendency agrees with the result of our previous Experiment 0 .

From these discussions, it can be concluded that the previous findings about the contribution of late sounds with a single directional energy component to $L E V$ are valid also when the late sounds have plural directional components.

\section{Contributions of directional late energy ratio and late- to-early energy ratio to $L E V$}

In Experiment 2, the degrees of contribution of the directional and total energy of late sound to $L E V$ are investigated, using the sound fields in which both $D L R$ and $T L L$ are varied.

\subsection{Experimental conditions}

The five loudspeakers for late sound were located at azimuth angles of $\pm 90^{\circ}$ (left and right), $0^{\circ}$ (front), $180^{\circ}$ (back) relative to the direct sound source on the horizontal plane, and at an elevation angle of $90^{\circ}$ above the listener (overhead). The $T L L$ value was varied over a range of -5 to $+5 \mathrm{~dB}$ corresponding to different levels of the total late energy, and the four DLRs were randomly varied from 0.05 to 0.81 . Thus, there were twenty different sound fields, as given in Table 3. All combinations of twenty stimuli, namely, one hundred and ninety pairs, were presented to fourteen subjects. Each subject judged each pair of stimuli four times, and thus, a total of 760 judgments were made.

\subsection{Results and discussion}

The psychological interval scale for $L E V$ was calculated from the experimental data. The results of conformity-tests with the Thurstone's Case V model showed that the experimental data was significant at a level below 5\%. The standard of judgment was agreed upon by all subjects at a level below $5 \%$ of significance.

In the same way as the preceding experiments, multiple

Table 3 Twenty sound fields used in Experiment 2.

\begin{tabular}{ccccccc}
\hline \multirow{2}{*}{$\begin{array}{c}\text { Stimulus } \\
\text { no. }\end{array}$} & \multirow{2}{*}{$(\mathrm{dB} P L$} & $T L L$ & \multicolumn{4}{c}{ Directional late energy ratios } \\
\cline { 4 - 7 } & $(\mathrm{dB})$ & $L E_{\text {late }}$ & $V E_{\text {late }}$ & $B E_{\text {late }}$ & $F E_{\text {late }}$ \\
\hline 1 & & 5 & 0.10 & 0.59 & 0.10 & 0.18 \\
2 & & 5 & 0.19 & 0.41 & 0.23 & 0.15 \\
3 & & 4 & 0.43 & 0.08 & 0.29 & 0.10 \\
4 & & 3 & 0.10 & 0.81 & 0.05 & 0.05 \\
5 & & 3 & 0.09 & 0.21 & 0.59 & 0.10 \\
6 & & 2 & 0.31 & 0.10 & 0.51 & 0.05 \\
7 & & 2 & 0.44 & 0.39 & 0.15 & 0.05 \\
8 & & 1 & 0.22 & 0.10 & 0.05 & 0.65 \\
9 & & 1 & 0.10 & 0.10 & 0.72 & 0.05 \\
10 & 63 & 0 & 0.32 & 0.19 & 0.10 & 0.41 \\
11 & & 0.18 & 0.69 & 0.05 & 0.05 \\
12 & & -1 & 0.09 & 0.32 & 0.21 & 0.41 \\
13 & & -1 & 0.65 & 0.29 & 0.05 & 0.05 \\
14 & & -2 & 0.26 & 0.29 & 0.28 & 0.09 \\
15 & & -2 & 0.20 & 0.55 & 0.15 & 0.05 \\
16 & & -3 & 0.17 & 0.16 & 0.55 & 0.05 \\
17 & & -3 & 0.15 & 0.54 & 0.09 & 0.20 \\
18 & & -4 & 0.10 & 0.42 & 0.40 & 0.11 \\
19 & & -5 & 0.60 & 0.20 & 0.11 & 0.10 \\
20 & & -5 & 0.76 & 0.11 & 0.06 & 0.06 \\
\hline
\end{tabular}

Table 4 The result of multiple regression analysis between $L E V$, and $T L L$ and three directional late energy ratios in Experiment 2, significant at $p<0.005$.

\begin{tabular}{ccccc}
\hline \multirow{2}{*}{$\begin{array}{c}\text { Multiple } \\
\text { correlation } \\
\text { coefficient }\end{array}$} & \multicolumn{4}{c}{ Standard regression coefficients } \\
\cline { 2 - 5 } & $T L L$ & $L E_{\text {late }}$ & $V E_{\text {late }}$ & $B E_{\text {late }}$ \\
\hline 0.932 & 0.967 & 0.630 & 0.223 & 0.393 \\
\hline
\end{tabular}

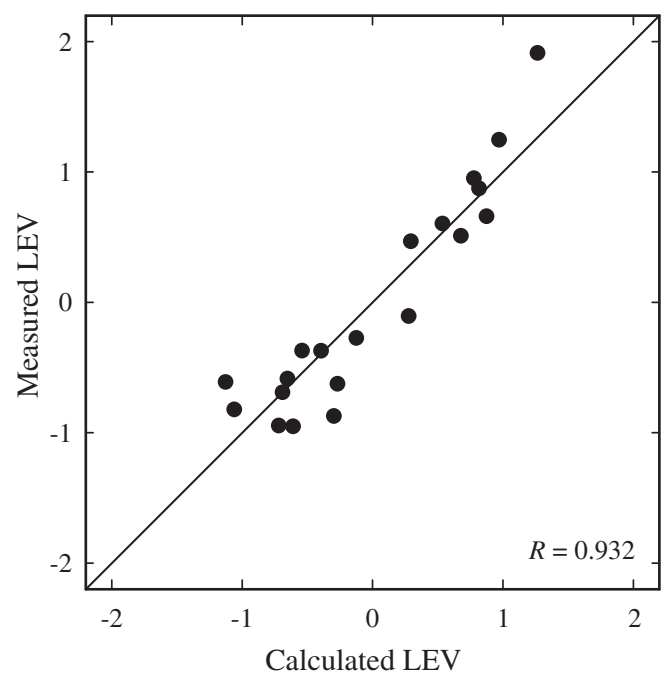

Fig. 4 Comparison between measured $L E V$ and calculated $L E V$ in Experiment 2: $R$, multiple correlation coefficient.

regression analysis was done to clarify the degrees of contribution of $D L R$ and $T L L$ to $L E V$. The psychological interval scale for $L E V$ was used as a criterion variable, and $T L L, L E_{\text {late }}, V E_{\text {late }}$, and $B E_{\text {late }}$ as explanatory variables. Table 4 shows the standard regression coefficients, which express the contribution of four physical parameters to $L E V$. A variance test ensured that the result was significant at a level below $0.5 \%$. Since the multiple correlation coefficient is 0.932 as shown in Fig. 4, the accuracy of this analysis is very satisfactory. The value of standard regression coefficient is the highest for TLL. Namely, this means that the contribution of the total late energy to $L E V$ is largest. Concerning the three $D L R$ s, the coefficient for $L E_{\text {late }}$ is the highest among them, and the coefficients for $V E_{\text {late }}$ and $B E_{\text {late }}$ are 35 and 62 percents of that for $L E_{\text {late, }}$, respectively. This result on $D L R$ agrees with the results of our previous experiments.

Finally, in order to confirm the validity of this result, the measured $L E V \mathrm{~s}$ in both Experiments 0 and 1, in which $T L L$ was constant, are correlated with the $L E V \mathrm{~s}$ calculated by the present regression equation. Table 5 shows the correlation coefficients between them. Good correlations are obtained, especially for $T L L$ values of +3 and $0 \mathrm{~dB}$, namely when the late energy is not smaller than the early one.

From these discussions, it can be concluded that the ratio of late energy to early one has the largest effect on perceived $L E V$. Furthermore, it is reconfirmed that $L E_{\text {late }}$ strongly affects 
Table 5 Correlations of calculated $L E V$ by the regression equation obtained in Experiment 2 with measured $L E V$ in Experiments 0 [2] and 1: $r$, correlation coefficient.

\begin{tabular}{|c|c|c|c|c|c|}
\hline \multirow{2}{*}{$\begin{array}{c}\text { Exp. } \\
\text { no. }\end{array}$} & \multicolumn{3}{|c|}{ Experiment 0} & \multicolumn{2}{|c|}{ Experiment 1} \\
\hline & (a) & (b) & (c) & (a) & (b) \\
\hline$T L L(\mathrm{~dB})$ & 3 & 0 & -3 & 3 & 0 \\
\hline$r$ & 0.956 & 0.946 & 0.610 & 0.974 & 0.934 \\
\hline
\end{tabular}

$L E V$, but $V E_{\text {late }}$ and $B E_{\text {late }}$ are also effective for $L E V$ at the rate of approximately thirty to sixty percents of the effect of $L E_{\text {late }}$.

\section{Conclusions}

Two kinds of psycho-acoustical experiments were conducted, and the relation between the directional and total energy of late sound and subjective listener envelopment was discussed. The results showed that: (1) The degree of contribution of late sound with a single directional energy component to $L E V$ is valid also when the late sound consists of plural directional components. (2) The total late energy affects $L E V$ most strongly when both $D L R$ and $T L L$ are varied in the sound fields. Furthermore, the present results definitively confirm previous findings that the late overhead and back energy ratios are also effective for $L E V$, though their effects are smaller than that of lateral energy ratio. These suggest that both the total late energy and DLR should be considered for the control of $L E V$ in concert halls.

\section{Acknowledgments}

A part of this research was supported by Grant-in-Aid for Scientific Research from Japan Society for the Promotion of Science (No. 15560521).

\section{References}

[1] H. Furuya, K. Fujimoto, Y. J. Choi and N. Higa, "Arrival direction of late sound and listener envelopment," Appl. Acoust., 62, 125-136 (2001).

[2] A. Wakuda, H. Furuya, K. Fujimoto, K. Isogai and K. Anai, "Effects of arrival direction of late sound on listener envelopment," Acoust. Sci. \& Tech., 24, 179-185 (2003).

[3] D. W. Robinson and L. S. Whittle, "The loudness of directional sound fields," Acustica, 10, 74-80 (1960). 